\title{
An Overview of the Main Topics and Applications of Graph Theoretic Problems in Multiphysics Engineering
}

\section{Garziad $M^{*}$ and Saka A}

Engineering Systems and Applications Laboratory, Sidi Mohamed Ben Abdellah Universiy, Fez, Morocco

\begin{abstract}
Graph-theoretic concepts are mostly used to study different applications in various areas such as electrical and mechanical engineering, generally multi-physics systems. The interest of researchers on this theory has importantly increased in the last decade. The main goal of this paper is to provide an overview of the use and applications of graph theoretic concept in modelling and analysis of systems in different fields. Therefore, the research topics discussed in this review are going to respect all the mentioned domains and the countries that apply this theory formulation in addition to the community of researchers who are identified by their publishers.
\end{abstract}

Keywords: Symbolic equations; Electrical system; Mechanical system; Vehicle dynamic; Multi-physics systems; Modeling simulation

\section{Introduction}

The physical modelling plays a significant role in representation and analysis of engineering systems. One of the important domains in physical modelling was the graph theoretic concept. This wellknown theory invented by the famous Swiss mathematician Leonhard Euler during 1707-1783, and it has been combined with principles of physics to develop algorithms for formulating the dynamic equations for multidomain systems. In literature, the first extensive development of systems started with the famous unsolved problem of his days; this issue was called "The Konigsberg Bridge Problem". Nevertheless, Euler in 1736 solved this problem by showing that it was impossible and laid the foundations of graph theory. In point of fact, the graph theoretical concepts are widely used to study and model various fields. They include computer science, electrical engineering, mechanical engineering and multibody system, flexible multibody, vehicle dynamics and multiphysics problems. This paper examines theoretic foundations and concepts of graph-theoretic modelling (GTM) to provide an overview of the use and applications of graph theoretic concept in modelling and analysis of systems in different fields. The paper is organised as follows: After this introduction, Section 2 gives a background of graph theory concept. Section 3 illustrates how graph theory is utilised in various domain of engineering applications.

Section 4 provides a statistical of paper related to graph theoretic published from 2000 to 2016. Last but not least, the review takes into consideration the research topics with referring to the discipline and the origins of their authors who worked in this theory formulation.

\section{Overview of Applications of Graph Theory}

\section{Definitions and terminology}

A graph $G$ is a pair $G=(V, E)$ consisting of a set $V$ of vertices and a set $\mathrm{E}$ of edges such that EVV. Graphs are amenable for a pictorial representation of a system using two basic components vertex and edges. It exists di erent methods of drawing the system graph and in the obtaining of the topological equations [1-5]. The vertex is represented by a dot while the edge is represented by a line segment connecting the dots associated with the edge. A graph is a set of connected lines. The lines represent symbolically the element of the system, the primary task of constructing a graph of the system is a simple line segment, the line segment is sometimes oriented to indicate the reference direction of element $e$ ort and ow variables in multiphysics modelling [6-8]. Graph- theoretical modelling represents physical systems using a set of nodes and connecting edges. In mechanical systems [9], the nodes represent reference frames and edges represent transformations between reference frames. In the electrical engineering [4], a circuit is drawn as a set of connected edges to forms a graph from which Kircho 's laws can be stated in concise algebraic form.

\section{Through and across variables}

The energy domain in which an edge exists dictates the physical meaning of it's through and across variables, as shown in Table 1. In the electrical domain, through variables are currents, and across variables are voltages. In the mechanical translational domain, through variables are forces and across variables are positions, velocities, and accelerations. In the mechanical rotational domain, through variables are moments and across variables are angles, angular velocities, and angular accelerations. In the hydraulic field, through variables are ow rate and across variables are pressure. In the thermal field, through variables are heat ow and across variables are Temperatures. The Across variables represent quantities measured by a device in parallel with the edge, whereas through variables represent quantities measured by a device in series with the edge.

\section{Numerical formulation vs. symbolical formulation}

A symbolical or numerical formulation approach can be used during the formulation stage to generate the governing equations that describe the system (Table 2).

On the one hand, the numerical approach will construct numerical matrices that represent the system at a given instant in time. These matrices are numerical by nature, and thus, they require to be reconstruct at each time step. On the other hand, a symbolical formulation method will produce the governing equations in the form of symbolic

${ }^{*}$ Corresponding author: Garziad M, Engineering Systems and Applications Laboratory, Sidi Mohamed Ben Abdellah Universiy, Fez, Morocco, Tel: +212 535609660; E-mail: mouad.garziad@usmba.ac.ma

Received July 12, 2017; Accepted August 28, 2017; Published August 31, 2017

Citation: Garziad M, Saka A (2017) An Overview of the Main Topics and Applications of Graph Theoretic Problems in Multiphysics Engineering. J App Mech Eng 6: 284. doi: 10.4172/2168-9873.1000284

Copyright: (c) 2017 Garziad M, et al. This is an open-access article distributed under the terms of the Creative Commons Attribution License, which permits unrestricted use, distribution, and reproduction in any medium, provided the original author and source are credited. 


\begin{tabular}{|c|c|c|c|c|}
\hline Physical Domains & Across Variables & Through Variables & Storage Elements \\
\hline Mechanical Rotational & Angular Velocity & Torque & Inertia \\
\hline Mechanical Translational & Translational Velocity & Rotational Velocity & Mass Spring \\
\hline Electrical & Voltage & Current & Capacitor, Inductor \\
\hline Hydraulic & Pressure & Flow Rate & Capacitance, Inertance \\
\hline Thermal & Temperature & Heat Flow & Resistance \\
\hline
\end{tabular}

Table 1: Analogies variables used in graph theory.

\begin{tabular}{|c|c|c|c|c|c|c|c|c|c|c|c|c|c|c|c|c|c|}
\hline Years & 00 & 01 & 02 & 03 & 04 & 05 & 06 & 07 & 08 & 09 & 10 & 11 & 12 & 13 & 14 & 15 & 16 \\
\hline Vehicle Dynamics & 1 & 1 & 3 & -- & -- & 2 & -- & 1 & 2 & -- & 1 & -- & 1 & -- & 1 & -- & 3 \\
\hline Flexible Multi-Body & 1 & 1 & 1 & - & -- & -- & -- & & -- & -- & -- & -- & -- & -- & -- & -- & -- \\
\hline Electrical Engineering & -- & -- & -- & -- & -- & 1 & -- & 1 & -- & 1 & -- & -- & 2 & 2 & 2 & 2 & 8 \\
\hline Mechatronics Engineering & 2 & 1 & 1 & 3 & 3 & 1 & 1 & 1 & -- & -- & -- & 1 & -- & -- & -- & 4 & 5 \\
\hline Mechanical Engineering & 1 & 1 & -- & -- & 2 & 1 & 4 & 1 & -- & -- & 1 & 1 & -- & 1 & 1 & -- & 4 \\
\hline
\end{tabular}

mathematical expressions that illustrate the system for all time. This later approach that is utilised for generating the system equations is based on relationships is denied by a graph. The formulation techniques connect the equations of the component with its topological equations obtained from the system graph to generate the symbolic equations.

\section{Research Topics}

The purpose of this section is to introduce the theoretical ideas of the graph in various kind of engineering like as electrical engineering, mechanical engineering, and multiphysics engineering. It also gives some important solved problems that are related to this concept. In the following subsections, the research topics will be described in more detail:

\section{Mechanical and multibody systems}

Graph-theoretical modelling (GTM) of mechanical systems is still a substantial problem, particularly, in the design of an appropriate model of multidimensional systems. The fundamental objectives of graph theoretic concept in mechanical context are the description of components and the interconnections between them, and also for selfformulation of governing equations. Graph theory is a powerful tool to describe a dynamic of multibody systems by automatic generations of motion equations. Different procedures exist in the review of the literature. The aim of them is to generate dynamic equation [5]. These equations are formulated typically by five simple steps. Graph-theoretical modelling for mechanical and multibody systems context draw a system using a set of nodes and connecting edges. Moreover, the nodes represent body fixed reference frames; then the edges represent all the physical entities of the system such as masses, forces, joints, and spring-damper connecting. The edges in the linear graph have a set of constitutive equations that represent the physical behaviour of the component. The multibody systems can be modelled using several coordinate schemes. The absolute coordinates are one of the easiest of choices. Nonetheless, the capabilities and the efficiency of the graph-theoretic method are not demonstrated by the absolute coordinate formulation. The general motion of any rigid body can be represented by the combination of purely translational and purely rotational motions. However, the combination of the translational and the rotational motion simultaneously show a problem in the modelling. In literature, the concept of graph theoretic was used to study some mechanical systems such as planar mechanical systems, a simple, double and triple pendulum which is driven by gravity, planar slidercrank, and Quick-Return mechanism. Moreover, the several symbolic and numeric formulations of this applications have been developed simultaneously a description of the systems components as well as the interconnections between them. Additionally, the production of these equations describes the motion of these systems. A multi-dimensional system in graph-theoretic modelling approach, based on VectorNetworks was introduced and investigated by Li [10]. This method employed a combination of topological equations obtained from linear graph theory and vector dynamics to formulate the governing equations of motion for multibody systems. In the twentieth century, many researchers in the multibody system such, Horace Trent [11], and others extended the graph theory to model mechanical systems by combining linear graphs with the set of constitutive equations that represent the physical behaviour of system components.

Dunlop and Khajepour [12] illustrated how graph theory was used in formulating and producing the dynamic equations of the drive belt pully mechanism. In fact, the system equations are formulated regarding hybrid branch-chord.

Banerjee and McPhee [13] introduced a new concept of modelling the multibody system by applying a graph-theoretic formulation to perform sensitivity analysis. In other words, they formulated a new method that contains all steps to generate an efficient sensitive equation for simulation of the pendulum. As a result, the developed formulation obtains both the sensitive equation and the ordinary equations.

Sherman [5] used a graph theoretic to develop a new methodology for modelling and analysing the gears, gear trains, and geared systems. He utilised the notion of cutset and circuit topologies for formulating the equations of motion of epicyclic gear systems.

Sreeram [14] presented a new technique to analyse and simulate the parametric design in torsional vibrating systems by using the graphtheory based technique, then the system's properties were modelled by a directed graph. The theory of qualitative and quantitative influences was also used to develop a technique which predicts the direction of change and accurate estimates of parametric values. This approach developed of graph theory was just a complementary to classical methods and conventional finite element method.

Venkata Rao [15] utilised a graph theory and matrix approach to illustrate a general method-ology for material selection for a given engineering component. The concept was developed by taking into account an index that evaluates the material for the component, also by considerate the material selection factors and their relative importance for the application.

Mathieu and McPhee [16] utilised the graph theory in order to represent and describe the topology of the mechanical system. In other words, he developed a new approach which is related to the Formulation 
and Selection coordinate based on a series of heuristics. The idea aimed to minimise the number of coordinates which produces models with faster simulation times. He proposed a method that applied on two mechanisms demonstrate the efficiency of it.

Joydeep Banerjee [17] focused on his research on using graphtheoretic formulations to develop an automated algorithm for the generation of sensitivity equations. He developed a software module which can derive the system equations and the sensitivity equations directly from the linear graph of the system. He also proposed an algorithm method that used direct differentiation to generate sensitivity equations at the component level and graph-theoretic methods to assemble the equation fragments to form the sensitivity equations.

Serge [9] used graph theory to model a mechanisms topology for a given mechanism. This approach concentrated on using a linear graph to de ne the coordinate set based on spanning tree. He developed two tree selections algorithm that enables to estimate the coordinate set that produces models having the fastest forward dynamic simulation times. Willem Peterson [18] provided a general overview of the application of graph theory in modelling multibody system.

Redmond and McPhee [19,20] introduced an automated formulation that models multibody kinematic and dynamic equations regarding indirect coordinates. The novel formulation was accomplished by collecting fundamental principles of classical mechanics with linear graph theory and the concept of a virtual joint between any two bodies. As a result, he illustrated the possibility of adding virtual joints to mechanisms modelled using a graph theoretical approach.

Uchida [21] demonstrated a new formulation for the automatic generation of systems of kinematic and dynamic equations using linear graph theory that fits real-time applications of several mechanical systems. The proposed approach targets the insertion of a technique that eliminates the Lagrange multipliers from the dynamic equations and obtains the ordinary differential equation for each independent acceleration.

Ling Xue [22] presented state of the art about the application of the graph theory in the representation of the modelling of a basic epicyclic gear train. Hence, they gave a review article about a method of kinematic and static force analysis that is based on the concept of fundamental circuit corresponding.

Banerjee and McPhee developed a formulation able to generate governing equation for dynamic systems in a flexible multibody. Further, they demonstrated through an example the applicability of graph-theoretic methods to represent and generate the dynamic and sensitivity equations for a given system.

Jain [23] used the concepts of graph theory to get a better understanding of the mathematical foundations of multibody dynamics. He shed light on the clarification of graph theoretic structural properties of spatial operator techniques in multibody dynamics In the end, he exploited these structural properties for developing a broad spectrum of analytical results and computational algorithms. Also, he introduced the notion of graph adjacency matrices and generalised it to de ne block-weighted adjacency matrices of the digraph adjacency matrices [24].

\section{Vehicle dynamics}

This subsection discusses and analyses the available literature and existing research in the vehicle dynamic field that is relevant to the current overview. In fact, there are only some few papers that use graph theory in the context of modelling and analysing the dynamic of the vehicle. One can consider the vehicles as a complex mechanical system where the edge can contain a set of constitutive equations that represent the physical behaviour of some component of the vehicle. This concept was used to represent some component of the vehicle, such as the torque converter for a Four-Wheeled vehicle and the tire and its interaction between the road in real time. The modelling of a full vehicle utilising Graph Theoretic Modelling was not yet treated regarding the complexity of modelling of the component of the vehicle, and also the energy ow exchanged between the different components. Consequently, it is a di cult task to apply the steps of generating the system of equations by employing GTM. The most problem of vehicle motion, especially the contact tire-road represents a subject of high interest to researchers because it plays an important part in safety [25].

William et al. [25] proposed a new method that enables to improve the computational efficiency and to analyse motion of wheels on three-dimensional roads. It was accomplished by creating a technique to combine tires using a linear graph that represents the interaction between tire and road. They formulated an approach that constructs the governing equations by using a symbolic formulation.

Schmitke et al. [26] combined graph theory and the principle of orthogonality to represent and to calculate the tire forces and moments. Moreover, the governing equations of the vehicle were generated with the symbolic formulation procedure based on linear graph theory. They integrated a tire model into the symbolic computer implementation which is used to create real-time simulations of vehicle dynamics. The tire component forms a list of symbolic expressions for significant tire variables, such as inclination and slip angle, etc.

Banerjee and McPhee [27] used a linear graph to model and to analyse the torque converter for an automotive. This component combines a hydraulical and mechanical field. Their goal was to generate a governing equation of torque converter in symbolic form, the system of equations simulated in a symbolical environment and compared with a conventional modelling method to demonstrate the efficiency of this concept.

Belkaloul [28] presented research that focuses on modelling of the vehicle with a trailer. So, he combined the method of vector network and the concept of a multibody system to model a vehicle trailer with six degrees of freedom and in the three-dimensional context. He developed, therefore, the differential equations that describe the motion of the vehicle.

Al-Hakim et al. [29] employed graph theory to represent a product and define the relationships between its components. They used the graph-theory concepts to visualise the energy flow and its application to design some components such as sliding gears, clutches, overrunning clutches, and fly wheels.

Vogt et al. [30] described a unified modelling of an electric vehicle utilising graph-theoretic modeling. The study was based on maple/dyna ex software for formulating and simplifying the governing equations of the electric vehicle.

Morency [31] studied a symbolic formulation procedure based on linear graph theory and the principle of orthogonality to generate governing equations for vehicle systems. They developed it into DynaFlexPro, which is a package that is known as DynaFlexPro/Tire. The tire component was used to simulate a vehicle dynamic. The aim of this development was to ensure a combination of DynaFlexPro and DynaFlexPro/Tire for constructing a model of any vehicle topology. 
They also de ned a list of intermediate variables that describe tire forces and moments as well as many other variables.

Reza and Murray [32] proposed a framework for formation stabilisation of multiple autonomous vehicles in a distributed fashion. Thus, they introduce a class of graphs that are more convenient for dealing with control of systems consisting of multiple agents. The goal was to find a distributed structural stabilisation of formations of multiple vehicles, regarding the state of the other vehicles, the graph theory was used to represent the multi-agent formations.

Reza and Murray [33] studied a three-dimensional analysis of vehicle stability using graph theory. Hence, they conducted their research in a semi-rigid vehicle supported by four tires in a planar curvilinear trajectory. Precisely, they proposed a direct coupling graph that represents the different forces and moments developed in contact road-tire and also vehicle body- tire. The lateral stability was evaluated through the static stability factor.

Reza and Murray [33] provided a graph theoretical framework to de ne formally the formations of multiple vehicles. Accordingly, they introduced new properties of minimally rigid graphs that compose the graph to smaller rigid subgraphs that help to construct a larger rigid graph, and they included a formal representation of split, rejoin, and recon guration maneuvers for formations of multi-vehicle systems. Additionally, they clarified the important role of graph rigidity and minimally rigid graphs in the construction of structural potential functions and manipulation of multiple formations.

Domotor [34] purposed a model that analyses the structure automotive supply chain networks by combining the theories of network and theories for traffic systems. The graph theory used to describe supply chain network where the nodes are suppliers, distribution centre nodes, warehouse nodes and the client node and the edges are representing the relationships between organisations.

Tanveer [35] presented the application of graph theory in modelling traffic controls problems in terms of vertices and edges. Particularly, he focused on minimising the waiting time of the traffic participants. The main idea was to determine the exact locations where the sensors have to be placed which minimises the total cost and the complete data of the traffic problem using the edges given by the edge connectivity and the vertices given by the vertex connectivity.

Laerriere et al. [36] illustrated a new method for decentralised stabilisation of vehicle formations using techniques from algebraic graph theory. The exchange of information of vehicles according to a pre-specified communication through a directed graph. In fact, they have demonstrated the close connection between spectral graph theory and one of the current methods of control of vehicle formations.

Gonzalez and Morgans [37] analysed and examined the stability of a formation of vehicles where the ow of information between vehicles can be changed drastically over time using the concept of graph theoretic.

Fax and Murray [38] introduced the notion of a communication graph. They proposed an averaging feedback law based on the ow of information. In fact, they combined graph theory and control theory to study stability criteria for vehicle formations. The interconnection between vehicles was modelled as a graph.

Cheng et al. [39] employed the graph theory to analyse and to study the efficiency of the power coupling mechanism for tracked vehicle transmission. Accordingly, they derived the system of equation using the graph theory.

\section{Flexible multibody}

The studies that have been achieved on the flexible multibody field combine a linear graph theory with the principle of virtual work. The main idea of graph theoretic in the flexible multibody field was about self-formulate and automatic generation of kinematic and dynamic equations. The dynamic systems were modelled by a linear graph in which nodes represent reference frames on rigid and flexible bodies; moreover, the edges illustrate the connection between components [40]. A notable feature of this formulation is that the users can choose the set of coordinates appearing in the equations of motion, by just selecting the spanning tree for the linear graph. This set can include absolute joint and elastic coordinates. The equations of motion are automatically generated regarding the corresponding branch coordinates. The graphtheoretic modelling (GTM) methods were extended to the analysis of some systems such as a thin flexible plate, Euler-Bernoulli beams, and planar four-bar mechanism. The first application of this approach in Flexible Multibody was created by Shi. Accordingly, they combined a linear graph theory with the principle of virtual work to obtain the dynamic formulation that describes the system. The system modelled by a set of nodes and edges, in which the nodes represent reference frames on flexible bodies, while edges represent components that connect these frames. Therefore, they used the DynaFlex package of Maple software to produce automatically the equation of motion for the planar slider-crank system. Richard et al. [40] treated the problem of modelling the flexible multibody systems by integrating the linear graph theory with the principle of virtual work and finite elements. Further, they studied thin exible plates for multibody systems.

Shi and McPhee [41,42] gave a new contribution in the studying and the development of the dynamic formulation. They also combined linear graph theory with kinematics and the principle of virtual work. As a result, a formulation has been implemented using maple software environment; which automatically generates the equations of motion for complex mechanical systems such as two-link and spatial flexible slider-crank.

\section{Electrical systems}

The application of graph theory for the electrical systems is still one of the most applications that are used in electrical network theory $[43,44]$. It has played a fundamental role in the examination and modelling the properties of the electrical network. The graph theory describes the electric network by drawing an equivalent graph that shows the network and solves some problems related to the electrical network by using a set of graphs, matrix theory, and Kirchho law. In this context, Kirchho has been the first one to use graph theory in electrical network theory and modelling. In fact, he employed graph theoretical concepts for characterising an electrical network. In other words, the electrical network is a collection of components such as resistors, inductors, capacitors, etc. The representation of the equivalent graph of the circuit network was one of the important tasks that study and analyse the electrical network. The electrical network in the theory of graph was formulated in terms of two variables, which are called current $i(t)$ and voltage $\mathrm{v}(\mathrm{t})$. The main goal of this approach was to generate a suitable mathematical model of the electrical network and to de ne the relations that govern the interaction of these elements. Physically speaking, the graph theoretic concept in network problem examines and predicts the behaviour of a system of interconnected physical elements regarding the characteristics of this latter. Too, it treats the way in which these elements are interconnected.

Holmgren [43] introduced the concept of graph theory in the 
analysis of electrical network and how the performance of the network can be a ected by the elimination of edge and vertice. The aim of his research was to model the electric power delivery networks as graphs. In fact, he concentrated on two power transmission namely Nordic and Western states.

Manjula introduced an essential introduction to graph theory application into network analysis. He gave a background on basic principles of electrical circuits to design a practical circuit using the concept graph theory. Additionally, he tried to show that solving some problems need to draw an equivalent linear graph [44].

Oepomo provided a methodology on how a graph theory and topology can be utilised to construct a Z-loop matrix. He took into consideration a faulted 3 phase power system. As a result, an algorithm construct Z-loop has been developed, that enables to construct Z-loop without generating loop incidence matrix for network circuit analysis [45].

Mezentsev [46] presented and developed a new automated discrete graph-theoretic model method for global mesh optimisation via nodal movement. In fact, the mesh is considered as an electric system. He suggested two main methods: the first one is to use a direct derivation of mesh optimisation model using primitives, and the second one was to employ multipole components. Both methods are based on a new correspondence between the structure of finite element stiffness matrix and the structure of the graph-theoretic M-matrix [47].

Berdewad and Deo [4] presented an overview of the application of the graph theory into a circuit network and how to draw electrical network by using this concept.

Felix et al. employed the concept of graph theory to compute and identify the contributing factors of individual generations and loads to the line flows appropriately. In fact, the vertices are used to model the system buses and the edges to represents the lines and transformers.

Werho et al. utilised the graph theory to analyze and to identify maximum ow transported within a directed graph in the network during real time [48].

Lanjewar et al. [49] developed a decision-making methodology by combining the graph theory and analytic hierarchy process methods to select and to evaluate the selection of energy technologies. The method proposes a graphical visualisation of different attributes and their interrelations in the form of a digraph model that helps to understand the complexity of the problem at a glance. Chitra and Nanjundappan employed the graph theory to identify the optimal power ow control of power transmission networks. A process of loop identification based on graph theory was utilised to ensure that each loop contains at least one active source [50].

Fax et al. in gave an overview of the methodology of graph theory matrix approach and its applications. More specifically, they presented the efficiency of graph theory matrix approach in various fields of science and technology [51].

Shafeeque et al. [52] presented a comparison of four loss allocation methods of graph theory based on loss allocation method over the other existing methods like proportional sharing, $\mathrm{Z}$ bus and modified $\mathrm{Z}$ bus loss allocation.

Bajpai et al [53] introduced a new methodology in the term to quantification resiliency and to maintain power supply to critical loads during extreme contingencies. The evaluation of the power distribution system has been de ned as a multi-criteria decision and its quantified using graph theoretic approach and Choquet integral [54].
Sun et al. [55] introduced a directed graph based method to mark carbon ow in power systems [56-83]. Abdullahi [79] focused on the application of graph theory in the electrical circuit. Specifically, he drew a circuit network in the concept of graph theory which facilitates the formulation of the adjacency and the incidence matrix by using matrix method.

Rajeswaran et al. [56] proposed a new approach for Phase Identification in Smart Grids Using Graph Theory and Principal Component Analysis in which the phase connectivity problem was solved.

Loh et al. [57] presented the concept of graph theory and their associated applications in di erents networking eld. As a matter of fact, the graph representation in technological fields are discussed and analyzed.

Elmqvist and Mattsson [58] developed a unified methodology to describe and analyze network topology. They utilized the linear graph to facilitate the modelling and the development of equations in symbolic form.

\section{Mechatronic systems}

Graph-theoretic modelling has been applied to the model in an easy way some mechatronic systems. In fact, this domain combined electrical, mechanical, and hydraulic systems. The components in this field can be represented by a single linear graph. Moreover, this approach facilitates the modelling of mechatronic multibody systems. The graph simplifies the understanding of the interactions between all the components within and across energy led cited above. Thus, the graph-theoretic formulation offers a significant flexibility concerning the systems modelling variables. The system of equations, which describes the multidisciplinary domain, can be generated easily with the application of graph theory formulation. Moreover, the system level dynamic equations can also be derived independently of the energy domains. So, the researchers of this domain have to think about two significant problems which are: considering independently the relation equations according to various subjects, and then coupling the variables belonging to

different fields. In fact, the model examines the behaviour of the component independently of how it is connected to the rest of the system. Concerning the mechatronic engineering, there are a lot of studies that have been conducted [59].

Han et al. illustrated the application of the linear graph to model a two-port element like an ideal transformer and gyrator. In actual of fact, they treated the representation the process of transmitting and converting energies between two different fields. Thus, the graph element includes a direct connection between the across and through variables that are associated with each branch of the graph. They also introduced methods to derive a set of state equations for some systems such as rack and pinions, ball screws, motors pump, compressor, etc.

Schmitke and McPhee [60] presented an extension of linear graphs to the modelling of mechatronic multibody systems employing the concept of graph theoretic. Consequently, they modelled mechatronic multibody systems by using the equivalent subsystem components. They focused on allowing a group of the component that contains internal degrees of freedom and/or internal constraints to be modelled as single components. In fact, they tried to facilitate the modelling of mechatronic multibody systems, furthermore, the coupled equations of the electrical and mechanical subsystems are self-formulated and self-generated in a symbolic form by using a package of maple known by Dyna ex. 
Scherrer and McPhee [61] in utilized the graph-theoretic to formulate and to model the electromechanical multibody systems. In fact, the electromechanical system is represented by a single linear graph that contain a set of constitutes equations. In other words, linear graph theory was used in order to generate the system of equations in the manner that is well-suited for computer implementation; the efficiency of this concept was demonstrated using two examples of electromechanical system.

Papadopoulos et al. [62] used linear graph theory to model an electro-hydraulic articulated forestry machine. The system includes both 1D (electrical, hydraulic, etc.) and 3D (mechanical) components. They developed different formulations that are used to generate the equations stemming from the $1 \mathrm{D}$ and $3 \mathrm{D}$ parts of the system.

Diaz et al. presented a new methodology for deriving the dynamic equations of mechatronic systems from component models. These components can be drawn by a single system graph. Moreover, the graph captures the interactions between all the components within and across energy domains - Rigid-Body mechanics, electrical, hydraulic, and signal domains, the dynamic system of equations can be derived independently from the underlying energy domains [63].

Schmitke et al. mentioned in their approach a unified formulation to generate systematically the governing equations of multi-domain systems in a symbolic form. They combined three concepts which are the principle of orthogonality, the principle of virtual work and virtual power. The formulation developed provides a significant flexibility with respect to the systems modelling variables. This permits the user to present the mechanical portion of the system using joint, absolute, absolute angular, or some hybrid set of coordinates.

Schmitke and McPhee [64] presented a procedure to model multibody systems using the subsystems method. This method applies to the extension of linear graph theory to produce the subsystem models.

McPhee et al. employed graph-theoretic modelling to present the system topology in order to obtain an e cient formulation for the system, they had integrated classical mechanics with linear graph theory and the concept of the virtual joint between any two bodies to formulate and generate multibody kinematic and dynamic constraint equations thereby allowing indirect coordinates. Special topologies like parallel manipulators were exploited by subsystem modelling approach.

Dao and McPhee [65] introduced a new modelling the electrochemical systems based on graph theory. They combined chemical, electrical, and thermo-dynamical processes. The goal behind this work was to employ a circuitry representation, branch and chord transformation techniques to generate automatically the dynamic equations of the system.

Desai [66] treated the fundamental issues underlying the control and coordination of multiple autonomous robots. In fact, they presented the problem of modelling a formation of nonholonomic mobile robots and developed a conceptual framework for transitioning from one formation to another. Also, they focused on the strategies for allowing con gurations of mobile robots using two methods, nonlinear control theory, and graph theory.

Reddy and Reddy [67] integrated the graph theory with the principles law of mechanics to study the dynamic modelling issue of the two-link robot arm driven by two DC motors. In fact, they represented the graph equivalent of the system, starting by the linear graph representation where the system of equations can be generated automatically.
Korres et al. [68] introduced numerical methods of topological observability analysis of a measured power system. In fact, the echelon form of a sparse rectangular test matrix used to determine topological observability, they also identified the maximal observable islands of a measured power system. The noniterative algorithm has been used to select the minimal set of pseudo-measurements which make the system observable. The theoretical background of the suggested algorithm is based on network graph properties.

Sass et al. [69] presented three different unified modelling of electromechanical particularly multibody systems based on the virtual work principle, linear graph and bond graph theories. The aim of their research is to show the advantages and limitations of physical modelling for a given system.

Rowell et al. [70,71] represented a modern control theory that helps in overcoming the limitations which existed in the classical control theory. As a matter of fact, he used an example of the physical system which is represented by RLC circuit by using linear graph method that generates the state space variables to write the differential equation between the input of the system and output variables.

Shai introduced a general approach for solving engineering problems by transforming them into other engineering fields through what is called graph representations. The method suggested can be implemented in the same way to both analysis and design problems. As a matter of fact, the graph representation can be utilised in various field as the mechanical system, electrical network and hydraulic network. The general approach that is employed in solving engineering problems by transforming engineering knowledge were adhered by four main steps.

Rowell [71] gave an approach to model Two-Port Energy Transducing elements using linear graph theory, in particular, they enumerated several steps that are derived from a set of state equations which includes transduction between di erent physical fields.

Kono and Katsura [72] examined the analysing of fundamental motion, they took into account the DOF of the system; the graph theory was used to identify the influence data in the motion. Therefore, an analysis of the removed data was conducted in the way to evaluate the eigenvector and maximum of eigenvalue from matrix adjacency.

Wang et al. [73] introduced a new class of the concept of importance measure that quantifies the importance of components in mechatronics systems. Precisely, they utilised the literature of mechatronic architecture and graph theory to de ne component network based on the notion of complex networks.

Banerjee and McPhee [74] presented a framework of graph theory to analyse and model the dynamic behaviour of an automotive hydrodynamic torque converter using graph theory method. The system comprises both hydraulic and mechanical components. Therefore, the graph theory used to capture the topology of the system and the interconnection of the constituent components. Finally, the model developed has been simulated and compared with the existing model in the literature.

Jayadev et al. [75] showed that the phase connectivity problem could be solved through a novel approach; the proposed formulation enables to use the Principal Component Analysis and its graph theoretic interpretation to infer the connectivity directly, they proposed an algorithm to infer phase connectivity from noisy measurements.

Liu et al. developed an optimal control to synchronise network 


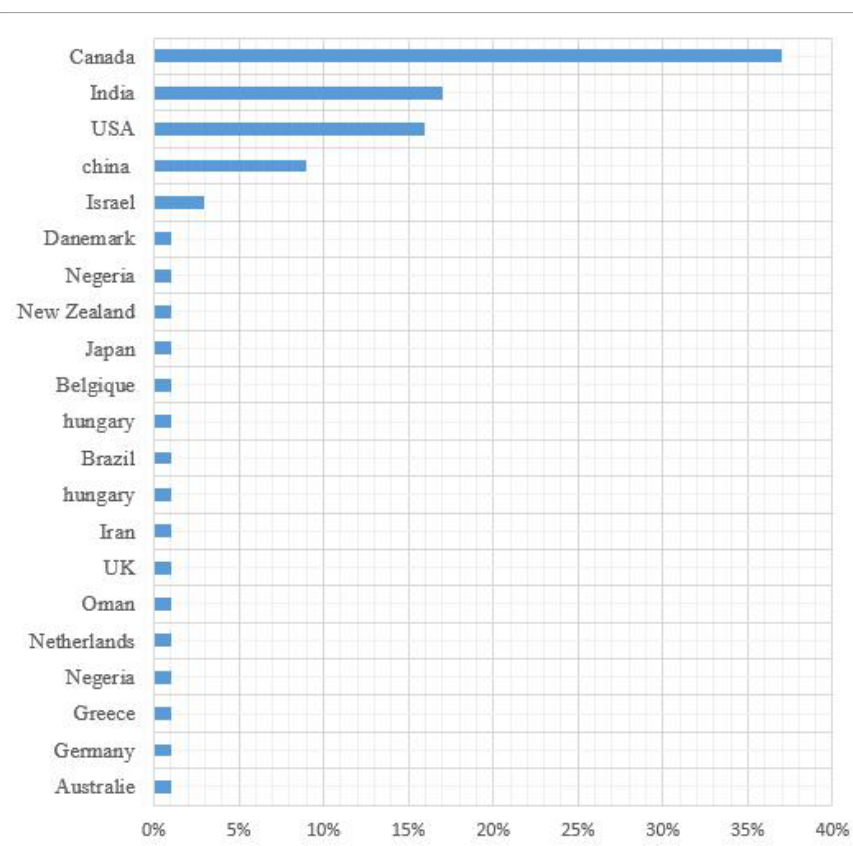

Figure 1: Region of papers.

multibody systems using the fundamental equation of mechanics. Hence, he used a new control law that is derived from the framework to achieve complete synchronisation of the network of Multibody Systems formulated with Lagrangian dynamics, the stability analysis performed by the algebraic graph theory.

Sheng et al. [76] used graph theory method to improve the precision of fault locations for CNC Machine Tools where system simplified as a fault mapping relationship between the system and the unit of the complex system and to identify the new fault with high speed.

\section{Discussion on Research Progress}

\section{Regions of origin of papers}

The 75 papers published are identified with regard to the 21 countries of the main authors too. The results are shown in the following figure. The countries with a larger contribution to the Journal are Canada, USA, India. This analysis might be change in future. The research topics published from 2000 to 2016 are analyzed. The following subsection contains the countries and the research topics that are related to this domain (Figure 1) [77-83].

\section{Research topic}

The seventy five papers published are identified with respect to the five main topics discussed in our second part. The results are presented in Table 2. Mechatronic engineering has been the most topics discussed. The difficult application is vehicle dynamics. Decreasing interest is observed in the more recent period for mechanical engineering and vehicle dynamics. It is noted that the interest of the authors concerning the application of this theory is developing in 2016; especially in the mechatronics engineering.

\section{Conclusion}

The aim of our paper is to present different research of graph theoretical modelling in various fields, especially in physical sciences as electrical engineering, mechanic and multibody system, flexible multibody, vehicle dynamics, and Multiphysics systems. Our article presented a general overview of the studies that have already been conducted in those fields. Therefore, it gave the localisation and number of publications of the previous articles starting from 2000 up to 2016 in the graph-theoretical concept. Further, we mentioned the principal ideas of this concept by using different fields that we have already cited above. The application of graph theoretical modelling approach in vehicle dynamics field is still one of the most critical and difficult topics that the researchers are working on currently.

\section{References}

1. McPhee $J$ (1998) Automatic generation of motion equations for planar mechanical systems using the new set of branch coordinates. Mechanism and Machine Theory 33: 805-823.

2. Muegge BJ (1996) Graph-theoretic modelling and simulation of planar mechatronic systems. Dissertation of University of Waterloo, Ontario, Canada.

3. Chou JCK, Singhal K, Kesavan HK (2005) Multi-body systems with open chains: Graph-theoretic models. Mechanism and machine theory 21: 273-284.

4. Berdewad O, Deo S (1996) Application of graph theory in electrical network Dissertation of University of Waterloo.

5. Lang SYT (2005) Graph-theoretic modelling of epicyclic gear systems Mechanism and Machine Theory 40: 511-529.

6. Diaz C, Christiaan A, Paredis JJ, Khosla PK (2000) Automatic generation of system-level dynamic equations for mechatronic systems. Computer-Aided Design 32: 339-354.

7. Stanisaw Z, Rysiski J (2016) Graph-based modelling in engineering.

8. Serge LM (2006) Automated selection of modelling coordinates for forward dynamic analysis of multibody systems.

9. Baciu G, Kesavan HK (1997) From particle-mass to multibody systems: graphtheoretic modeling. IEEE Transactions on Systems, Man, and Cybernetics-Part A: Systems and Humans 27: 244-250.

10. Tai-Wai L, Andrews GC (1986) Application of the vector-network method to constrained mechanical systems. J Mechanisms, Transmissions, and Automation in Design 108: 471-480.

11. Horace TM (1955) Isomorphisms between oriented linear graphs and lumped physical systems. The J Acoustical Society of America 27: 500-527.

12. Dunlop J, Khajepour A (2000) A new multi-terminal pulley model for use in graph theoretic modelling. Mechanism and machine theory 35: 1601-1621.

13. Banerjee JM, McPhee JJ (2014) Graph-theoretic sensitivity analysis of multibody systems. J Comp and Nonlinear Dynamics 9: 041009.

14. Sreeram TR (2005) Graph theory based parametric influences applied to torsional vibration analysis. Advances in Engineering Software 36: 209-224.

15. Venkata Rao R (2006) A material selection model using graph theory and matrix approach. Mater Sci and Eng 431: 248-255.

16. Lger M, McPhee J (2007) Selection of modeling coordinates for forward dynamic multibody simulations. Multibody System Dynamics18: 277-297.

17. Baneerjee J (2013) Graph-theoretic sensitivity analysis of dynamic systems. University of Waterloo, Ontario, Canada.

18. Petersen W (2013) A volumetric contact model for planetary rover wheel/soi interaction. University of Waterloo, Ontario, Canada.

19. Koenig HE, Blackwell WA (1960) Linear graph theory-A fundamental engineering discipline. Transactions on Education 3: 42-49.

20. McPhee J, Redmond SM (2006) Modelling multibody systems with indirect coordinates. Comp Methods in Appl Mech and Eng 195: 6942-6957.

21. Uchida TK (2011) Real-time dynamic simulation of constrained multibody systems using symbolic computation. University of Waterloo, Ontario, Canada.

22. Hui-Ling X, Geng L, Yang XH (2015) A review of graph theory application research in gears. Proceedings of the Institution of Mechanical Engineers, Part C: J Mech Eng Sci: 0954406215583321.

23. Jain A (2011) Graph theoretic foundations of multibody dynamics. Multibody System Dynamics 26: 335-365. 
24. Jun L, Ji J, Zhou J (2016) Synchronization of networked multibody systems using fundamental equation of mechanics. Appl Math and Mech 37: 555-572.

25. Bombardier W, McPhee J, Chad S (2010) Symbolic Formulation of multi-body dynamic equations for wheeled vehicle systems on three-dimensional roads. SAE Int J Mater and Manufact 3: 454-467.

26. Schmitke C, Morency K, McPhee J (2008) Using graph theory and symbolic computing to generate e cient models for multi-body vehicle dynamics. Proceedings of the Institution of Mechanical Engineers, Part K: J Multi-body Dynamics 222: 339-352.

27. Banerjee JM, McPhee J (2012) Graph-theoretic modeling and dynamic simulation of an automotive torque converter. Proceedings of the 7th Vienna International Conference on Mathematical Modelling.

28. Belkaloul A (2012) Modlisation des systmes multi-corps rigides base sur la mthode des rseaux virtuels. Universit Laval.

29. Al-Hakim L, Kusiak A, Mathew J (2000) A graph-theoretic approach to conceptual design with functional perspectives. Computer-Aided Design 32 : 867-875.

30. Vogt HS, Schmitke CC, Jalali K, McPhee JJ (2008) Unified modelling and realtime simulation of an electric vehicle. Int $\mathrm{J}$ Vehicle Autonomous Systems 6 : 288-307.

31. Morency K (2007) Automatic generation of real-time simulation code for vehicle dynamics using linear graph theory and symbolic computing. University of Waterloo, Ontario, Canada.

32. Olfati-Saber R, Murray RM (2002) Graph rigidity and distributed formation stabilization of multi-vehicle systems. Decision and Control. IEEE.

33. Olfati-Saber R, Murray RM (2017) Three-dimensional analysis of vehicle stability using graph theory. Graph-Based Modelling in Engineering. Springer International Publishing, Berlin. p. 117-129.

34. Domotor A, Peter T, Szabo K (2016) Mathematical modeling of automotive supply chain networks. Periodica Polytechnica Transportation Engineering 44: 181-186.

35. Tanveer S (2016) Application of graph theory in representing and modelling traffic control problems. Int Math and Comp Appl Res (IJMCAR): 2249-6955.

36. Lafferriere G, Williams A, Caughman J, Veerman JJP (2005) Decentralized control of vehicle formations. Systems and Control Letters 54: 899-910.

37. Gonzalez C, Morgansen KA (2005) Stabilization of dynamic vehicle formation configurations using graph Laplacians. Proceedings 38: 121-126.

38. Fax JA, Murray RM (2002) Graph laplacians and stabilization of vehicle formations. IFAC Proceedings Volumes 35: 55-60.

39. Cheng D, LiuY, Qin D, Hu M, Wan WF (2017) Analysis of the efficiency of the power coupling mechanism for tracked vehicle transmission based on the graph theory.

40. Richard MJ, Bouazara M, Therien JN (2011) Analysis of multibody systems with flexible plates using variational graph-theoretic methods. Multibody System Dynamics 25: 43-63.

41. Pengfei S, McPhee J (2002) Symbolic programming of a graph-theoretic approach to flexible multibody dynamics. Taylor \& Francis: 123-154.

42. Shi P, McPhee J (2000) Dynamics of flexible multibody systems using virtual work and linear graph theory. Multibody System Dynamics 4: 355-381.

43. Holmgren AJ (2006) Quantitative vulnerability analysis of electric powe networks. KTH.

44. Manjula V (2010) Electrical networks by graph theory. Int J of Math and Eng IEEE 149: 1368 -1373.

45. Oepomo TS (2008) A step-by-step method for z-loop construction using graph theory and topology for power system studies. Computational Technologies in Electrical and Electronics Engineering. IEEE Region 8 Int Conf on IEEE.

46. Mezentsev AA (2004) A generalized graph-theoretic mesh optimization model. IMR 255-264.

47. Wu FF, Yixin N, Wei P (2000) Power transfer allocation for open access using graph theory-fundamentals and applications in systems without loop flow. IEEE transactions on power systems 15: 923-929.

48. Werho T, Vittal V, Kolluri S, Wong SM (2016) Power system connectivity monitoring using a graph theory network flow algorithm. IEEE.
49. Lanjewar P, Rao R, Kale A, Taler J, Oclon P (2006) Evaluation and selection of energy technologies using an integrated graph theory and analytic hierarchy process methods. Decision Science Letters. 5: 237-348.

50. Chitra Su, Nanjundappan D (2014) Optimal power OW control of powe transmission networks using graph algorithms. J Vibration and Control 21 3320-3327.

51. Fax, JA, Murray RM (2016) Graph laplacians and stabilization of vehicle formations. Indian J Sci and Tech.

52. Shafeeque AK, Karthikeyan SP, Sahoo SK (2016) Comparison of graph theory approach with other methods on transmission loss allocation problem in deregulated electricity market. Applied Mechanics and Materials. Trans Tech Publications.

53. Bajpai P, Chanda S, Srivastava AK (2016) A Novel metric to quantify and enable resilient distribution system using graph theory and choquet Integral. IEEE Transactions on Smart Grid.

54. Shai O (2003) Transforming engineering problems through graph representations. Advanced Engineering Informatics 17: 77-93.

55. Sun T, Feng D, Ding T, Chen L, You S (2016) Directed graph based carbon ow tracing for demand side carbon obligation allocation. In Power and Energy Society General Meeting (PESGM) IEEE.

56. Rajeswaran A, Narasimhan S (2015) Network topology Identification using PCA and its graph theoretic interpretations.

57. Loh SL, Gan CK, Cheong TH, Salleh T (2016) An overview on network diagrams: graph-based Representation. J Telecommunication, Electronic and Comp Eng (JTEC) 8: 83-86.

58. Elmqvist H, Mattsson SE (2016) Exploiting model graph analysis for simplified modeling and improved diagnostics. Proceedings of the 7th International Workshop on Equation-Based Object-Oriented Modeling Languages and Tools ACM: 7-14.

59. Pradeep S, Christian LH, Paredis JJ, Khosla K (2005) Object-oriented libraries of physical components in simulation and design. Simulation.

60. Schmitke C, McPhee J (2005) Forming equivalent subsystem components to facilitate the modelling of mechatronic multibody systems. Multibody System Dynamics 14: 81-110.

61. Scherrer M, McPhee J (2003) Dynamic modelling of electromechanical multibody systems. Multibody System Dynamics 9: 87-115

62. Papadopoulos E, Mu B, Frenette R (1997) Modeling and identification of an electrohydraulic articulated forestry machine. Int J Multiscale Comp Eng. IEEE International Conference 1: 60-65.

63. Schmitke C, McPhee J (1997) A procedure for modeling multibody systems using subsystem models. Robotics and Automation 1: 2 .

64. McPhee J, Schmitke C, Redmond S (2004) Dynamic modelling of mechatronic multi-body systems with symbolic computing and linear graph theory. Mathematical and Computer Modelling 10: 1-23.

65. Dao TS, McPhee J (2011) Dynamic modeling of electrochemical systems using linear graph theory. J Power Sources 196: 10442-10454.

66. Desai JP, Ostrowski JP, Kumar V (2001) Modeling and control of formations of nonholonomic mobile robots. EEE transactions on Robotics and Automation 17: 905-908.

67. Reddy RB, Reddy CA (2003) Dynamic modeling of two-link robot arm driven by DC motors using linear graph theory and principles of mechanics. National Conference on Trends in Mechanical Engineering, USA. p. 130-132.

68. Korres GN, Katsikas PJ, Clements KA, Davis PW (2003) Numerical observability analysis based on network graph theory. IEEE Transactions on Power Systems 18: $1035-1045$.

69. Sass L, McPhee J, Schmitke C, Fisette P, Grenier D (2004) A comparison of different methods for modelling electromechanical multibody systems. Multibody System Dynamics 12: 209-250.

70. Rowell D (2002) State-space representation of LTI systems.

71. Rowell D (2004) Linear graph modeling: two-port energy transducing elements. Massachusetts Institute of Technology Department of Mechanical Engineering Boston, UK.

72. Kono T, Katsura S (2016) Clarification of fundamental motion using hierarchica clustering and graph theory. IEEJ J Industry Applications 5: 108-116. 
Citation: Garziad M, Saka A (2017) An Overview of the Main Topics and Applications of Graph Theoretic Problems in Multiphysics Engineering. J Appl Mech Eng 6: 284. doi: 10.4172/2168-9873.1000284

73. Wang Y, Bi L, Li SL, Hao MS (2017) A complex network-based importance measure for mechatronics systems. Physica A: Statistical Mechanics and its Applications 466: 180-198.

74. Banerjee J, McPhee J (2016) System dynamic modelling and simulation of hydrodynamic machines. Mathematical and Computer Modelling of Dynamical Systems 22: 54-86

75. Jayadev P, Bhatt S, Nirav P, Pasumarthy R (2015) A novel approach for phase identification in smart grids using graph theory and principal component analysis, Cornell University Library, Ithaca, NY, USA.

76. Sheng B, Deng C, Wang Y, Xie S (2016) Improved multi-faults diagnosis for CNC machine tools. Mechatronic and Embedded Systems and Applications (MESA), 12th IEEE/ASME International Conference on IEEE 1-6, Budapest, Hungary.

77. Deo N (2016) Graph theory with applications to engineering and computer science. Prentice Hall Series in Automatic Computation, USA
78. Banerjee J, McPhee J (2017) Graph-theoretic modelling and sensitivity analysis of dynamic systems. Graph-based modelling in engineering. Springer International Publishing, Berlin. p. 107-116.

79. Abdullahi S (2014) An application of graph theory to the electrical circuit using matrix method 10: 164-166.

80. Rajeswaran A, Bhatt NP, Pasumarthy R (2016) A novel approach for phase identification in smart grids using graph theory and principal component analysis. American Control Conference (ACC), USA.

81. Morency KW (2007) Automatic generation of real-time simulation code fo vehicle dynamics using linear graph theory and symbolic Computing. Waterloo, Ontario, Canada.

82. Paredis HL, Christiaan JJ, Khosla K, Pradeep K (2001) Object-oriented libraries of physical components in simulation and design. Waterloo, Ontario, Canada.

83. Wellstead PE (1979) Introduction to physical systems modellin 